\title{
A Triaxial Flexible Magnetic Tunnel Junction Sensor for Catheter Tracking
}

\author{
Y. Mashraei ${ }^{1,2}$, L. Swanepoel ${ }^{1}$ and J. Kosel ${ }^{1}$ \\ ${ }^{1}$ Computer, Electrical and Mathematical Sciences and Engineering Division \\ King Abdullah University of Science and Technology (KAUST), Thwual, Saudi Arabia \\ ${ }^{2}$ King Abdulaziz City for Science and Technology (KACST), Riyadh, Saudi Arabia
}

\begin{abstract}
A cardiac catheterization procedure is a minimally-invasive surgery, which is employed in case of various cardiovascular conditions and requires a high precision. The method relies heavily on $x$-ray imaging and contrast dyes to guide the catheter inside the blood vessels, posing risks to patients and the medical staff. Thus, a miniaturized tri-axes magnetic sensor is developed for orientation monitoring using the Earth's magnetic field, to assist the guiding of the catheter. The magnetic sensors are realized by flexible magnetic tunnel junctions on ultra-thin silicon substrate, ensuring a high sensitivity to magnetic fields. These flexible sensors have a bending endurance of over 1000 cycles without signs of fatigue. Three sensors are mounted on the tip of a catheter, implementing a sensor-on-tube concept for orthogonal 3-axes measurements. The sensors have an MR ratio of $29 \%$ and a high sensitivity of about $9 \Omega /{ }^{\circ}$. With a weight of only $16 \mu \mathrm{g}$ and a thickness of $4 \mu \mathrm{m}$, each sensor adds a negligible weight and increase in size, making them attractive for applications, where extreme miniaturization is sought. The flexible tri-axes MTJ is mounted onto the tip of a cardiac catheter with $3 \mathrm{~mm}$ in diameter.
\end{abstract}

Keywords - catheterization; triaxial sensor; tunnel magnetoresistance; magnetic tunnel junction;

\section{INTRODUCTION}

Surgical tool development is an accelerating field, especially in the case of minimally invasive surgery, where small incisions are used to insert tubes and surgical instruments [14]. To perform the procedure, $\mathrm{x}$-ray images together with contrast agents are used to visualize the blood vessels and track the orientation and location of the catheter. Obviously, $\mathrm{X}$-ray exposure is harmful to the patients as well as the medical staff [5-7]. The excessive use of the contrast dye poses a serious risk of contrast medium-induced nephropathy, which has been reported to have increased to $30 \%$ in patients with congestive heart failure [8-10]. A prime drive for the development of alternative navigation solutions is to reduce $\mathrm{x}$ ray exposure, while providing an accurate estimation of the catheter's orientation during the procedure [11-14].

In this work, we propose a highly miniaturized tri-axes sensor, based on magnetic tunnel junctions (MTJ). In order to maintain a high performance, the MTJ sensors are first fabricated on a standard Si wafer, which is then thinned down $[15,16]$. The substrate becomes mechanically flexible and can conform to the curvature of the catheter. The tri-axes magnetic sensor exploits the Earth's magnetic field to track the orientation of the catheter's tip. The vector summation of the magnetic projections (or alternatively $\mathrm{Mx}, \mathrm{My}$ and $\mathrm{Mz}$ projections) points toward the magnetic north, which can be used as a reference direction to track the tip's orientation.

\section{METHODS}

The triaxial sensor consists of 3 flexible MTJ sensors that are placed on the perimeter of the catheter, in a way that aligns their sensitive axes effectively perpendicular to each other, as illustrated in Fig.1.

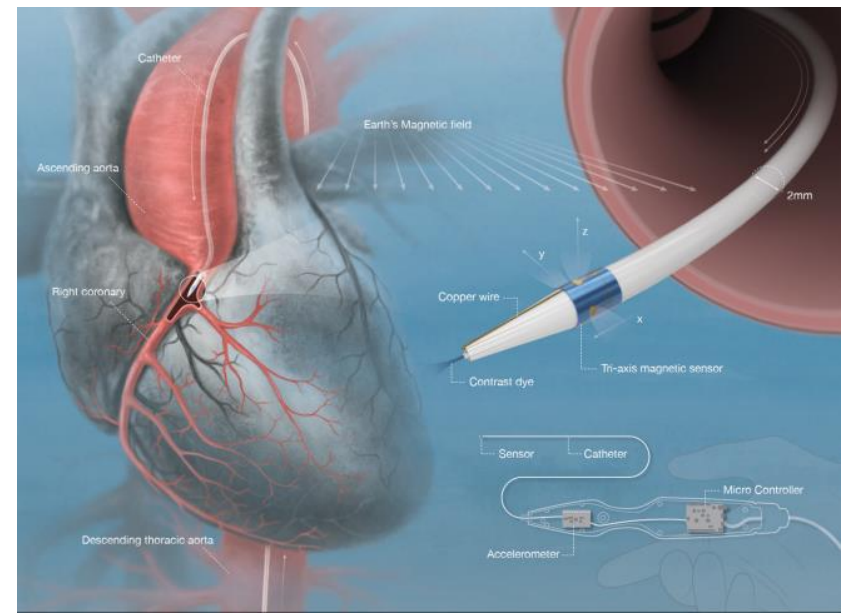

Fig. 1. The catheter's tracking system with a tri-axes MTJ sensor on the tip and wired to the handle at the catheter's end "Image by Ivan Gromicho, KAUST".

A $\mu$-controller-based tracking system (Arduino Nano) was developed that derives the Earth's magnetic field components MX, MY and MZ from the tri-axes sensor. The sensor is mounted to the catheter's tip and wired to the $\mu$-controller outside the body via wires inside of the catheter. Fabrication And Characterization Setups

The MTJ sensor fabrication process is described elsewhere [15]. The tri-axes MTJ sensor exploits the shape of the catheter to create three sensors with perpendicularly aligned sensitive directions. They are placed on a hollow acrylic tube as a carrier, instead of attaching it directly onto the catheter. 
The tube is laser cut to have a $2 \mathrm{~mm}$ diameter and a shell thickness of $0.5 \mathrm{~mm}$. It has four cut-through small openings $(0.1 \mathrm{~mm})$ for copper wires to go through. The wires are then soldered directly onto the contacts of the flexible sensors at low temperature. Once the sensors are placed and soldered, a heat shrink tube is used to secure the assembly and protect it. The assembly is shown in Fig. 3.
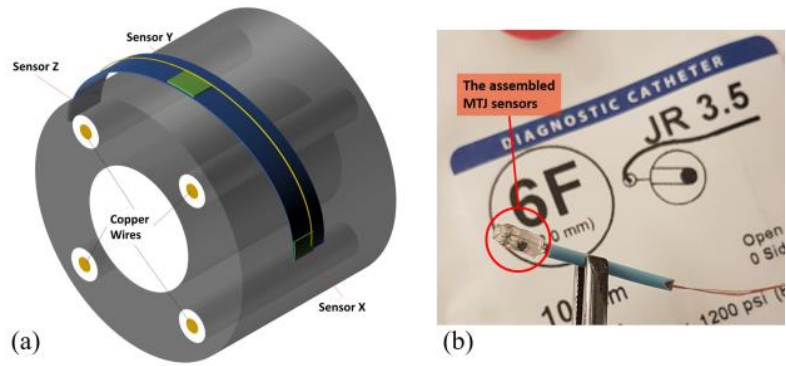

(b)

Fig. 3. (a) Illustration of the carrier tube used for the assembly. (b) Assembled triaxial sensor.

\section{RESULTS AND DISCUSSIONS}

First, the flexible MTJ sensor showed a sensitivity of 1.4 $\mathrm{k} \Omega / \mathrm{Oe}$ and a resistance of $29 \mathrm{k} \Omega$, as depicted in Fig.4a. The angular response in Fig. $4 \mathrm{~b}$ is sinusoidal with a maximum change of $15 \%$ change, corresponding to $3.8 \mathrm{k} \Omega$, and a sensitivity of $8.85 \Omega /{ }^{\circ}$.
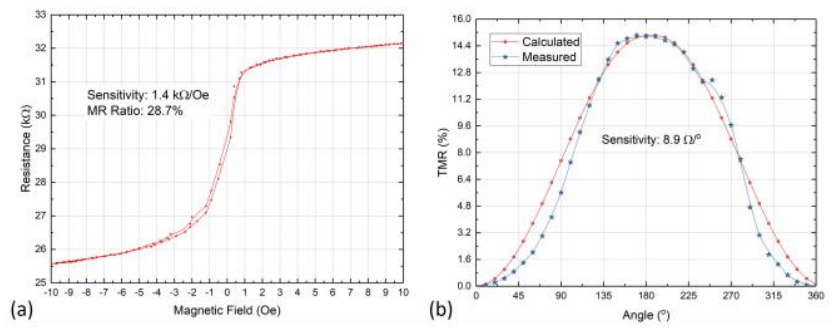

Fig. 4. (a) MTJ characteristic curve and (b) the angular response.

The rotation of the tri-axes sensor about its three axes and the magnetic projections MX, MY and MZ are depicted in Fig. 5.

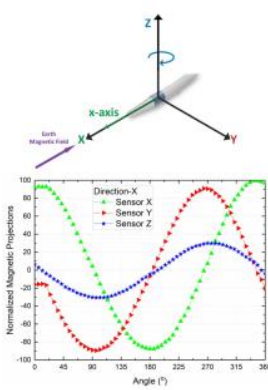

Direction $\mathrm{X}$

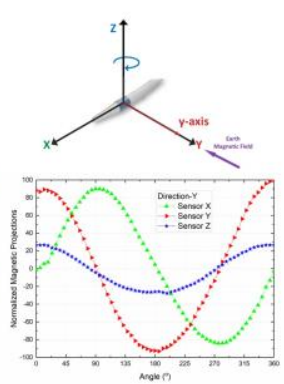

Direction $Y$

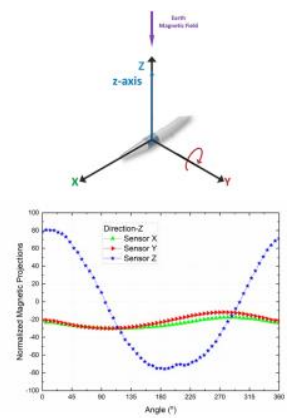

Direction Z
Fig. 5. Angular response of the triaxial sensor obtained simultaneously.
In direction-X, the Sensor $\mathrm{X}$ " which corresponds to the $\mathrm{X}$ axis sensor" showed a good response with minimum value along the Earth's magnetic field, and maximum when it aligns antiparallel to it. The response of the Sensor $\mathrm{Y}$ "which corresponds to the y-axis sensor" was shifted by $90^{\circ}$ from Sensor X, which indicates good alignment of these two sensors to each other. However, Sensor Z "which corresponds to the $\mathrm{Z}$ axis sensor" showed a considerable cross sensitivity with Sensor Y. In Direction-Y, the same behavior was observed, and the cross sensitivity of Sensor $Z$ became evident. The Sensor $\mathrm{Z}$ was the only sensitive sensor to $\mathrm{Z}$-direction, but the MZ projection was not aligned perfectly with the Earth's magnetic field, since the maximum and minimum values do not coincide with this alignment. These results indicate the misalignment of Sensor Z during the assembly, which induced considerable cross-sensitivity between Sensor Z and Sensor Y. This misalignment is a result of the manual mounting process, with which it is difficult to get an precise placement and orientation of the sensor dies.

\section{REFERENCES}

[1] S. Stampfl et al., "Initial experience with a new distal intermediate and aspiration catheter in the treatment of acute ischemic stroke: clinical safety and efficacy," Journal of neurointerventional surgery, pp. neurintsurg-2015-011801, 2015.

[2] T. M. Bashore et al., "American College of Cardiology/Society for Cardiac Angiography and Interventions Clinical Expert Consensus Document on Cardiac Catheterization Laboratory Standards: A report of the American College of Cardiology Task Force on Clinical Expert Consensus Documents endorsed by the American Heart Association and the Diagnostic and Interventional Catheterization Committee of the Council on Clinical Cardiology of the AHA," Journal of the American College of Cardiology, vol. 37, no. 8, pp. 2170-2214, 2001.

[3] H. Calkins et al., "Hrs/ehra/ecas Expert Consensus Statement on Catheter and Surgical Ablation of Atrial Fibrillation: Recommendations for Personnel, Policy, Procedures and Follow-up: A report of the Heart Rhythm Society (hrs) Task Force on Catheter and Surgical Ablation of Atrial Fibrillation developed in partnership with the European Heart Rhythm Association (ehra) and the European Cardiac Arrhythmia Society (ecas); in collaboration with the American College of Cardiology (acc), American Heart Association (aha), and the Society of Thoracic Surgeons (sts). Endorsed and Approved by the governing bodies of the American College of Cardiology, the American Heart Association, the European Cardiac Arrhythmia Society, the European Heart Rhythm Association, the Society of Thoracic Surgeons, and the Heart Rhythm Society," Europace, vol. 9, no. 6, pp. 335-379, 2007.

[4] C. A. Warnes et al., "ACC/AHA 2008 guidelines for the management of adults with congenital heart disease: a report of the American college of cardiology/American heart association task force on practice guidelines (writing committee to develop guidelines on the management of adults with congenital heart disease) developed in collaboration with the american society of echocardiography, heart rhythm society, international society for adult congenital heart disease, society for cardiovascular angiography and interventions, and society of thoracic surgeons," Journal of the American College of Cardiology, vol. 52, no. 23, pp. e143-e263, 2008. 
[5] R. W. Harbron et al., "Cancer incidence among children and young adults who have undergone $\mathrm{x}$-ray guided cardiac catheterization procedures," European journal of epidemiology, vol. 33, no. 4, pp. 393-401, 2018

[6] S. McFadden, R. Mooney, and P. Shepherd, "X-ray dose and associated risks from radiofrequency catheter ablation procedures," The British journal of radiology, vol. 75, no. 891, pp. 253-265, 2002.

[7] L. K. Wagner, P. J. Eifel, and R. A. Geise, "Potential biological effects following high X-ray dose interventional procedures," Journal of Vascular and Interventional Radiology, vol. 5, no. 1, pp. 71-84, 1994.

[8] M. Tepel, P. Aspelin, and N. Lameire, "Contrast-induced nephropathy: a clinical and evidence-based approach," Circulation, vol. 113, no. 14, pp. 1799-1806, 2006.

[9] H. S. Thomsen and S. K. Morcos, "Risk of contrast-mediuminduced nephropathy in high-risk patients undergoing MDCT-a pooled analysis of two randomized trials," European radiology, vol. 19, no. 4, p. 891, 2009.

[10] G. Marenzi et al., "Comparison of two hemofiltration protocols for prevention of contrast-induced nephropathy in high-risk patients," The American journal of medicine, vol. 119, no. 2, pp. 155-162, 2006.
[11] D. Filgueiras-Rama et al., "Remote magnetic navigation for accurate, real-time catheter positioning and ablation in cardiac electrophysiology procedures," Journal of visualized experiments: JoVE, no. 74, 2013.

[12] L. Di Biase et al., "Remote magnetic navigation: human experience in pulmonary vein ablation," Journal of the American College of Cardiology, vol. 50, no. 9, pp. 868-874, 2007.

[13] K. Totsu, Y. Haga, and M. Esashi, "Three-axis magnetoimpedance effect sensor system for detecting position and orientation of catheter tip," Sensors and Actuators A: physical, vol. 111, no. 2-3, pp. 304-309, 2004.

[14] B. Li, M. N. Kavaldzhiev, and J. Kosel, "Flexible magnetoimpedance sensor," Journal of Magnetism and Magnetic Materials, vol. 378, pp. 499-505, 2015.

[15] S. Amara et al., "High - Performance Flexible Magnetic Tunnel Junctions for Smart Miniaturized Instruments," Advanced Engineering Materials, vol. 20, no. 10, p. 1800471, 2018.

[16] L. M. Loong et al., "Flexible $\mathrm{MgO}$ barrier magnetic tunnel junctions," Advanced Materials, vol. 28, no. 25, pp. 4983-4990, 2016. 